\title{
Urban Energy Reform Drives Urban Economic Development
}

\author{
Ying Su \\ North China Electric Power University \\ 2585467578@qq.com
}

Keywords: Economic Development; Urban Energy; Energy Reform; Urban economy

\begin{abstract}
With the deepening of global energy reform, build a global energy Internet, promote the clean and green way to meet the global power needs, has gained more and more international recognition. Through energy change to drive the development of urban economy, give full play to the advantages of both, to maximize the cleaning alternative of production side and the electricity replacement of consumption side, so that the impact of energy reform achieve the international leading level to provide comprehensive economic development ideas.
\end{abstract}

\section{Current Situation of China's Urban Energy System}

The urban energy system is a complicated network system in which energy resources are developed, transformed, transported and consumed, and so on. It is closely related to social, economic, policy and environmental factors, showing mutual influence and the interaction. At the same time, the complex interaction between the various parts of the energy system also leads to the existence of a large number of uncertain information, directly or indirectly affect the formation of energy planning programs and energy decision making. The urban energy system is a large system composed of secondary energy users who are acquired, processed and converted by secondary energy, delivery and distribution in the city in the original form.

Urban energy resource consumption is large, the over-exploitation of fossil fuels and the over-reliance on them brought about a serious shortage of energy resources, energy supply and demand gap intensified, energy external dependence is too high, increased energy insecurity, air pollution, excessive greenhouse gas emissions, deterioration of urban living environment and a series of serious problems. On the one hand, electricity production, oil refining, coking, steel and other industrial activities as well as motor vehicles to discharge large amounts of air pollutants, which is far beyond the environmental carrying capacity, so the city was subjected to a wide range of serious acid rain pollution and haze pollution. The main reason for the large-scale haze pollution in the whole city is that the coal-based energy structure and the proportion of direct coal are too high.

On the other hand, high temperature and low temperature and other extreme weather struck in recent years, energy supply and demand contradictions intensified. The peak of the summer (winter) during the electricity shortage, coal shortage, oil shortage, gas shortage and other energy crisis events are common, seriously affecting the urban industrial production and residents living. Energy security issues have become increasingly prominent. This series of serious problems is the essence of China's energy resources endowment "inherent deficiencies", energy development mode of "acquired disorders" contradictions of the long-term accumulation and concentrated exposure [1]. The structure of China's urban energy system network diagram is the integration of three subsystems, primary energy supply system, energy processing conversion and distribution systems and terminal energy consumption system. In the urban energy system, the focus of energy supply is security, involving the self-sufficiency of energy resources, energy reserves, changes in energy demand and other factors [2].

The Energy Development Strategy Action Plan (2014-2020) issued by the General Office of the State Council in 2014 clearly stated, vigorously improve energy efficiency, reasonable control total energy consumption, and actively develop natural gas, nuclear power, renewable energy and other clean energy, reduce the proportion of coal consumption, continuously promote energy structure optimization, strengthen the development and implementation of energy development strategies, plans, policies and 
standards, strengthen energy regulation, speed up the pace of power system reform, promote direct trade between supply and demand sides, build a competitive electricity trading market. To improve the situation of the "tight" environment and "hard" energy supply, optimize the energy structure, solve the contradictions between rapid development of urban and energy shortages, environmental pollution, coordinate the relationship between urbanization process and rational utilization of energy resources, promote the energy consumption revolution, supply revolution, technological revolution and institutional revolution, achieve the sustainable development of the city.

\section{Energy Structure Reform Objectives}

In the national contribution target after 2020, China has proposed to reach $\mathrm{CO} 2$ emissions peak around 2030 and early to reach the peak. By 2030, CO2 emissions per unit of GDP were 60-65\% lower than in 2005, non-fossil energy in the primary energy consumption in the proportion increased to $20 \%$ around.

The current stage of industrialization in China, industrial terminal energy consumption and $\mathrm{CO} 2$ emissions accounted for $70 \%$ of the total energy consumption and $\mathrm{CO} 2$ emissions, while the developed countries generally only about $30 \%$, is an important area of energy saving and carbon reduction in China. China promotes industrial restructuring and transformation through the development of strategic emerging industries and modern service industries, at the same time, eliminate lagging production capacity, improve energy efficiency. By 2020, CO2 emissions from the industrial sector are expected to peak, CO2 emissions per unit of industrial added value will fall by more than $50 \%$ in 2005, can reduce the energy consumption intensity of the industrial sector higher than the national energy consumption intensity decline rate and lay the foundation for achieving national goals.

Table 1 CO2 emission intensity

\begin{tabular}{|l|l|}
\hline CO2 emissions & Compared to 2005 \\
\hline CO2 emissions per unit of GDP in 2030 & Fall 60-65\% \\
\hline CO2 emissions per unit of industrial added value in 2020 & Fall more than 50\% \\
\hline
\end{tabular}

China should vigorously develop new energy and renewable energy to achieve the target of non-fossil energy accounted for $6.8 \%$ in 2005 to $20 \%$ in 2030. It is an important support to achieve the goal of reducing CO2 intensity and emission targets. By 2030, the proportion of coal fall from $70 \%$ in 2005 to $50 \%$ or less, coupled with an increase in the proportion of natural gas, $\mathrm{CO} 2$ energy per unit of energy consumption can be reduced by $20 \%$. At that time, non-fossil energy supply will reach about 1.2 billion tons of standard coal which is equal to the sum of total energy consumption in Japan, France and the UK, and non-fossil energy installed capacity will reach about 1.3 billion kilowatts which is equal to the total installed capacity of the United States. In addition to that wind power and solar power installed capacity will reach 3-4 million kilowatts, hydropower installed capacity of 4-5 billion kilowatts, nuclear power installed capacity of about 150 million kilowatts.

Table 2 Various energy installed capacity

\begin{tabular}{|l|l|}
\hline Target of 2030 & Compared to 2005 \\
\hline Non-fossil energy installed capacity & 1.3 billion kilowatts \\
\hline Wind power and solar power installed capacity & $3-4$ million kilowatts \\
\hline Hydropower installed capacity & $4-5$ million kilowatts \\
\hline Nuclear power installed capacity & 1.5 billion kilowatts \\
\hline
\end{tabular}

\section{Future Energy Development Trends}

State Grid Energy Research Institute chief expert Feng Qing dong commented that micro-grid is the future development trend, the basis of energy Internet and the basic unit of active distribution network, We will accept renewable energy as much as possible, reduce the cost of micro-grid, improve energy 
efficiency, and coordination with the distribution network to achieve through the dynamic energy management system for remote visualization of energy equipment operations and intelligent control. The new energy micro-grid represents the future energy development trend, which is important measures of implement energy production and consumption revolution, apart from this, it is the important carrier of promote energy development and management change and an innovative application in the field of energy of "Internet+", It is of great significance to promote energy conservation and energy conservation and sustainable development. Simultaneously, the new energy micro-grid is a concrete way of releasing the grid side to the social main body and it is in line with the direction of power system reform as well as create a huge space for the new energy development.

\section{An Analysis of the Factors Affecting Urban Development}

The "Soft Factors" Affecting Urban Development. Four types of soft factors influencing urban development include human capital, digital information, civilized culture and ecological environment, they have an important impact on urban development. High-quality human resources and investment can make the city industry get access to resources and product competitive advantage, making the city more attractive. Modern cities are based on communication, computer and networked information resources, extensively use digital information processing technology and network communication technology. The city's various information resources to be integrated to form a new urban planning, construction and management of the concept and control means has become increasingly important. Civilized culture also has an important impact on reflect the characteristics of urban culture, achieve architectural style and natural environment coordination, cultural traditions and the characteristics of the times blend, local characteristics and cultural common harmony, shaping the image of urban culture, create a city cultural atmosphere, enhance the cultural quality of cities and cities, Cultural heritage and improve the quality of the humanities, and promote the healthy and healthy development of the city. The optimization of the ecological environment has a positive and indispensable role in the harmony between man and nature. Therefore, we must take into account the four "soft factors" in order to achieve the optimal development of the city [3].

The Effect of Urban Energy Reform on Urban Development. The specific effects of urban energy reform on urban development include four aspects. First of all, urban energy reform can make rational use of urban spatial structure. Secondly, this is conducive to improving the city's competitiveness. Thirdly, it is conducive to the city to achieve economies of scale. Lastly, land resources will be reasonably used. There are three main aspects of urban energy reform measures to promote urban development. Firstly, strengthen the management of urban energy reform. Secondly, improve the scientific and seriousness of urban energy reform. Lastly, ensure the effective implementation of urban energy reform [4].

The Role of Government Policy in the Development of Resource-Based Cities. The country's macroeconomic policy directly determines the rise and fall of China's resource-based cities. In the "balanced layout", "construction of three lines" policy background, China's Midwest formed a large number of energy, raw materials and other basic industries as the pillar of the resource-based city. Due to the lack of extensive economic ties and obviously geographical advantages in the resource-based cities, they are exposed to the free and open economic conditions. The current dilemma of resource-based cities is closely related to the economic policies of the national planning period. After the reform and opening up, due to the prevalence of "market failure" in resource-based industries, the externalities of resource extraction and the disorderly market structure make the loss of resource-based cities unable to be compensated by the perfect market. So the government should give full play to the functions of macro-control to support the sustainable development of resource-based cities. Resource-based urban transformation requires the support of the government, the development of slanted industrial policy, to give financial subsidies, through the improvement of infrastructure, comprehensive development of regional economy and other measures to achieve the revival of the recession. As the development of economy, we should strengthen the concern of social issues, set the economic and social development as a revitalization of resource-based city's goal [5]. 
The Effect of Energy Consumption on Urban Development. The level of urban development has a two-way impact on energy consumption. Generally speaking, the higher the level of urban development, the higher the demand for energy consumption, so the greater the importance of energy supply. Simultaneously, in the case of energy supply constraints, the energy consumption structure is irrational, which in turn will inhibit the urban development process. In the stage of rapid urban development, energy consumption rigid demand is relatively large. At this stage, if the policy in energy consumption and urban development deviates shift, it will lead to inefficient allocation of large-scale resources and cause obstacles to future urban development [6].

The Role of Energy Internet in Urban Development. To develop a smart city, we must first solve the problem of urban energy consumption and emissions [7]. Energy internet can provide various services, for example, urban charging pile operation, enterprise energy monitoring, the overall and fixed-point analysis and consulting, so that it can meet the business, government multi-energy management and regulatory needs. Energy internet brings low-carbon, energy saving, environmental protection, green, healthy and sustainable is precisely the important part of building the wisdom city. So the energy internet and the wisdom city is an inseparable community which is mutual influence, mutual promotion and common development. The mainly contribution of energy internet to the wisdom city is that it can accelerate the transformation of economic development model, achieve the healthy development of energy conservation industry, create new industry and accelerate the transformation of economic structure, therefore provide a solid and reliable energy base for the city[8]. The construction of energy internet has also strengthened the use of high-tech electronic products and new electrification equipment in its industry and promote the development of smart grid. Through various technologies conversion and application in the specific enterprise production, such as electricity instead of coal and the coal to the oil, gradually increase the proportion of electricity energy in the terminal energy consumption and promote the in-depth development of the wisdom city [9]. The construction and development of the energy internet enhance the level of information city, at the same time, promote the "triple play" development and actively build a number of urban public network platform. Not only that the construction of energy internet can also improve the social environment and production environment, improve the utilization of energy resources, and to a large extent reduce energy consumption and reduce carbon emissions. Energy internet support for the wisdom city is mainly reflected in elevating urban green development, achieving urban electricity's safe and reliable, build the city's nervous system and promote the development of related enterprises, besides enrich the connotation of urban services and so on [10]. Energy internet show its own value in the economy, energy, people's livelihood and other aspects as well as support the construction of intelligent city in life, in production and other fields.

\section{Conclusion}

Energy reform implements the optimization of various energy resources with a strong and reliable, cost-effective, clean and environmentally friendly, flexible and interactive, friendly and open, healthy and sustainable performance, meets the wisdom city's energy needs and promote the development of all aspects of the city eventually.

\section{References}

[1] Ya Zhou: Improved Fuzzy Programming Method and Urban Energy System Management (MS., North China Electric Power University, China 2015).

[2] T.Q. Sun, Yi. Zheng, Hao Wang and X .L .Wu: China Population Resources and Environment, 2010 No.11, p.1.

[3] L.C. Zhang: The South of China Today, 2010 No.12, p.102.

[4] Song. Yang: Architectural Knowledge, 2016.

[5] X.M. Wu and Ying. Yang: Economic Review, 2004 No.3, p.66.

[6] X.F. Liu: Comprehensive Evaluation and Coordination Analysis of Urbanization and Energy Consumption in Shandong Province (MS., China Ocean University, China 2013). 
[7] S.Y. Zhang and A .K .Miao: China Electric Power, 2016 No.3, p.12.

[8] T.T. Ha, Y.J. Zhang and V.V. Thang: Clean Energy, Vol. 5 (2017) No.3, p.389.

[9] D.M. OJEDA, R.G. RUBIO and A. VARGAS: Clean Energy, Vol. 5 (2017) No.3, p.299.

[10] C. HE, T.Q. LIU and M. SHAHIDEHPOUR: Clean Energy, Vol. 5 (2017) No.3, p.375. 